\title{
Mapping Urban Accessibility in Data Scarce Contexts Using Space Syntax and Location-Based Methods
}

\author{
Jose Morales ${ }^{1} \cdot$ Johannes Flacke ${ }^{1}$. \\ Javier Morales ${ }^{1} \cdot$ Jaap Zevenbergen ${ }^{1}$
}

Received: 5 January 2017 / Accepted: 11 September 2017 /

Published online: 2 October 2017

(C) The Author(s) 2017. This article is an open access publication

\begin{abstract}
Data scarcity is still a common barrier to adequately understanding urban access in Global South countries. Widely used location-based methods address the traditional definition of accessibility as the easiness to reach land-uses by means of available mobility modes. Space Syntax instead analyses accessibility as network centrality focusing only on the topological and geometric properties of urban layouts, making it comparatively less data-intense. However, the interpretation of its outputs is limited to its own theory. Knowledge is missing on how such metrics are comparable to the metrics produced by location-based methods. The objective of the research was to compare both approaches for mapping urban accessibility in two cities in Guatemala. Our hypothesis tested the assumption that Space Syntax metrics could consistently reflect accessibility conditions that so far have only been measured by location-based methods. We proposed an approach using volunteered geo-information and produced accessibility maps following both approaches that were then compared using Pearson correlations. Space Syntax metrics at low and high radii are consistently correlated with location-based access to land uses that reflect location quality at neighbourhood and city-wide scale correspondingly. Space Syntax metrics at lower radii reflect time-based access restrictions either posed in the location-based analyses or by reduced accessibility by public transport. The hypothesis acceptance, $p<0.01$, expands the scope of accessibility knowledge derivable from limited data availability using Space Syntax, which is relevant for its applicability in data-scarce contexts by planners and researchers in the Global South. Rather than replacing location-based methods Space Syntax offers an important complementary measure to geographical accessibility. This having been said, Space Syntax could contribute to early-stage planning by gaining overall insights into patterns of urban access.
\end{abstract}

Jose Morales

j.a.morales@utwente.nl

1 Faculty of Geo-Information Science and Earth Observation (ITC), University of Twente, P.O. Box 217, 7500 AE Enschede, The Netherlands 
Keywords Urban accessibility - Space syntax - Location-based - Guatemala ·

Data scarcity. VGI

\section{Introduction}

Understanding urban accessibility is fundamental for land use and transport planning (Curtis and Scheurer 2010; Geurs and Van Wee 2004; Curl et al. 2011), as it is one of the key aspects for agglomeration economies, economic growth, and quality of life (Ahlström et al. 2011; Kourtit et al. 2015; Rietveld 2015). Two conceptions of urban accessibility can be distinguished. Geographic accessibility is the most common one and is defined as the opportunity at origin to reach a destination, or vice-versa, given the impedance between both locations (Curl et al. 2011; Handy and Niemeier 1997; Batty 2009; Geurs and Van Wee 2004; Ingram 1971; Albacete et al. 2015). The combined effect of land use distribution and infrastructure components at a given location determines geographic accessibility (Geurs and van Eck 2001). Geometric or general accessibility, on the other hand, is concerned with network centrality and focuses on the topological, metric and geometric properties of urban layouts (Webster 2010; Hillier et al. 2010; Bafna 2003; Batty 2004).

Two methodological approaches correspond to the two concepts of access. Locationbased measurements have been the preferred methods to analyse geographic accessibility (Geurs and van Eck 2001; Curl et al. 2011). In turn, Space Syntax (SSx) is a set of theories and methods with long-standing development whose purpose is to analyse geometric accessibility (Webster 2010; Karimi 2012; Hillier et al. 1976). The availability of geographic data (e.g. land use, road and public transport networks), the easiness of interpretation and applicability of geographic information systems (GIS) have facilitated implementing location-based methods for transport planning purposes. However, the scarcity of official data and capacities for processing the same is still an important barrier in Global South countries (Yeh 1991; Yeh 1999; Ahlström et al. 2011) such as Guatemala. Common problems are incomplete or outdated data sets as resources might not be available for periodic collection and maintenance.

Alternative sources of information such as volunteered geographical information (VGI) might be potentially useful when dealing with scarcity of official data (Arsanjani et al. 2015), jointly with considering a geometric accessibility concept. The SSx method is less data-intense than traditional location-based methods. Only a representation of a roads network is needed for the analysis. Previous work has already reported associations between SSx metrics with relevant urban phenomena: flows of people (Hajrasouliha and Yin 2015), land use and construction density (Kim and Sohn 2002; Hillier et al. 2000; van Nes et al. 2011; Hillier et al. 2010) and real estate values (Matthews and Turnbull 2007; Netzell 2012).

SSx has also been debated regarding its dual analytical approach (Ratti 2004; Hillier and Penn 2004; Porta et al. 2006). Batty (2013) emphasized the problem of mathematically relating the topological-based measurements with the intuitive geographic ones (e.g. distance or time) and proposed an analytical framework to reconcile SSx with metric information. The SSx approach has attempted to prove itself a complementary tool to aid planners and researchers in accessibility studies, particularly in data-scarce contexts. However, the interpretation of its outputs remains limited to its own theory 
and knowledge is missing on how such metrics are comparable to the measurements produced by location-based methods. These observations restrict its applicability as an analytical approach when data availability is limited.

The objective of this research was to compare a geographical and a geometrical approach for mapping urban accessibility. Our hypothesis tested the assumption that Space Syntax metrics could consistently reflect urban access conditions that so far have only been measured by location-based methods. By testing this hypothesis we attempted to contribute in empirically bridging both approaches and expanding the scope of knowledge derivable from SSx. This is relevant for planning practice as regards the applicability of available methods to address accessibility-related planning tasks in the context of Global South cities with data-challenging environments. Two cities in Guatemala were studied in order to examine the applicability of both approaches in different heterogeneous and fragmented contexts. We developed a methodological framework for analysing accessibility using SSx and location-based methods. This included a tailored approach that uses VGI to mitigate unavailability of official data. We computed locationbased access per mode of transport to key land uses that are relevant in planning practice and are commonly associated with urban-economic dynamics. We further derived two SSx metrics at the road-level at various spatial scales. Finally, the results from both approaches were compared using Pearson correlation. The strength and significance $(p<0.01)$ were evaluated. We elaborated on how geometric accessibility measurements provided information that was comparable to geographic access to various land-uses per mode of transport, its limitations and its applicability in practice.

The remainder of this paper is organised as follows: section 2 introduces the location-based and SSx-based accessibility measurements used in this research. Section 3 describes the methodological framework and introduces the case study areas. Section 4 presents the results and discussion. Finally, section 5 addresses the conclusions.

\section{Location-Based Methods and Space Syntax}

\section{Location-Based Methods}

Location-based methods are widely used in research and practice (Koenig 1980; Wegener and Fürst 2004; Handy and Niemeier 1997; Geurs and Van Wee 2004; Albacete et al. 2015). They aim to analyse accessibility considering four components (Geurs and van Eck 2001, page 35): (1) mobility infrastructure (i.e. roads, public space, public transport), (2) land-use location, (3) temporal conditions of the previous two, such as variability of travel-times and available land uses during the course of the day or week and (4) personal-level characteristics and restrictions. A plausible accessibility model would attempt to address these aspects as far as possible in accordance with its purpose. However, it will be limited by the availability of geographic data.

Three commonly used location-based measurements are: (1) impedance to closest facility, (2) cumulative opportunity, and (3) potential accessibility. The first analyses proximity following the criteria of shortest trip where impedance is commonly defined by travel time (per mobility mode), distance or cost. Cumulative opportunity measures 
the number of reachable attractions within a given impedance threshold and takes the form of Eq. 1.

$$
A_{i}=\left\{\begin{array}{c}
\Sigma M_{j}, \text { if } d_{i j} \leq R \\
0, \text { if } d_{i j}>R
\end{array}\right\}
$$

Where $A$ is the access at origin $i ; M$ is the size of the attraction at destination $j ; d$ is the impedance between $i$ and $j$; and $R$ is the radius restriction. The potential accessibility can be traced back to Stewart and Warntz (1958) and Hansen (1959). It accounts for the size of attraction (e.g. number of jobs) and the effect of distance on the interaction probability between origin and destination. Such effect is commonly named distance decay. The measurement takes the form of Eq. 2.

$$
A_{i}=\sum M_{j} \alpha \exp \left(-\beta^{*} d_{i j}\right)
$$

Where $M$ is equal to the size of the attraction at $j$; and $\alpha$ and $\beta$ are constant parameters that determine the distance decay.

These three measurements are simple and less data-intense compared to other location-based measurements such as those based on balancing factors and derived from time-space geography (Geurs and van Eck 2001; Curl et al. 2011). The components of Eqs. 1 and 2 can be adapted to data availability. For example, impedance can be measured in planar or network distance, time, or cost. Although planar or even network distance could be used if data is scarce, real mobility conditions are represented better when using travel time or cost per mode of transport. The size of attraction ' $M$ ' in both equations could simply represent the number of facilities available (e.g. number of public spaces). Even though a more realistic representation could for instance to include floor area.

Limitations of these measurements have been described by Geurs and van Eck (2001). A cumulative opportunity does not distinguish impedance or attraction size differences between the various destinations reached within the fixed threshold. These limitations are overcome by the potential accessibility measurement. However, decay parameters should be calibrated per mobility mode and trip purpose, which is more data-demanding. Results are less intuitive to interpret, although acceptable to non-specialists. Some drawbacks of the potential accessibility measurement are: influence of self-potential, attraction within origin zone; no distinction between matching types of attraction and individual preferences; only addressing of the spatial distribution of attraction supply, not the demand of those. Extensions of the basic gravity model have addressed these drawbacks at a cost of more data needs and interpretability.

\section{Space Syntax (SSx)}

SSx is a network analytical formalism to analyse a type of accessibility that also has an economic significance (Webster 2010). Hillier et al. (1993) describe this 
access type as the easiness to move through and to places given the spatial arrangement of urban layouts, which has shown to be correlated with flows and attraction of movement. Urban economies are tightly linked to these dynamics as certain land uses benefit from these flows based on a maximum profitability rationale (Hillier 1996). Positive correlations between SSx with real estate values and construction density support such a relation (Matthews and Turnbull 2007; Kim and Sohn 2002; Netzell 2012). It follows that we can expect a positive correlation between accessibility as analysed in SSx and location-based accessibility to various land uses that follow an economic rationale, or service type of activities where the purpose is to be reachable.

Sharing similar grounds with SSx is the Multiple Centrality Assessment (MCA) method (Porta et al. 2005). The main difference between the two is that SSx analyses are computed using a dual graph, while MCA is based on a primal one. Opposite to the dual graph, in the primal approach intersections are treated as nodes and streets as edges. While SSx is known for pioneering in the studies of network centrality applied to cities, MCA presents itself as an enhanced method with recent evidence of its capacity to correlate with location of economic activities (Porta et al. 2010; Porta et al. 2012). However, in our research we consider it appropriate to implement the SSx approach as it benefits from a notably larger body of literature empirically supporting its applicability in various urban studies, in planning and design processes and with respect to the availability of applications for direct implementation within GIS. Following Law (2017), metrics analysed at the street level via a dual approach would be adequate to compare with accessibility metrics that are derived from travel times and location of places along the street, not at street-junctions.

SSx analyses over road centre-lines are done using a segment angular analysis (SAA) technique (Turner 2007; Hillier and Iida 2005). It is a geometric weighting method that works as an impedance parameter based on the idea that persons seek to minimize their angular deviation when choosing trip routes (Dalton 2003). Implicitly SSA accounts for the continuity of road segments, but without incurring in an explicit generalization process (network simplification) such as the "street-name approach" or the "continuity negotiation algorithm" (Jiang and Claramunt 2002; Porta et al. 2006).

Two main variables are analysed: integration and choice. Integration is equivalent to network closeness, and choice to network betweenness (Freeman 1977; Porta et al. 2005). Integration measures how close each segment is to any other segment in the network. Choice measures the cumulative number of times that each segment is used in shortest trips from every segment towards every other segment. Impedance in SAA is based on angular deviation between segments, unlike the measurements of time or distance in geographic access. Thus, angular integration at any given $x$ segment takes the form of Eq. 3,

$$
\operatorname{Int}_{\theta}(x)=\left(\sum_{i=1}^{n} D_{\theta}(x, i)\right)^{-1}
$$


where $n$ is equal to the number of segments in the system, and $D_{\theta}(x, i)$ is the angular depth between $x$ segment and any other segment in the network, $i$. Depth indicates the cumulative angular deviation. Angular choice is expressed in Eq. 4,

$$
C h_{\theta}(x)=\frac{\sum_{i=1}^{n} \sum_{j=1}^{n} \sigma(i, x, j)}{(n-1)(n-2) / 2}
$$

where $\sigma(i, x, j)=$ ' 1 ', once $x$ is used to go from $i$ to $j$, else $=$ ' 0 ' and being $i \neq x \neq j$. Hillier et al. (2012) suggest a normalization procedure for integration (NAIN) and choice $(\mathrm{NACH})$ to a scale ranging from -3 to 3 . While the normalizing choice is highly recommended, normalising both values allows comparing the results between segments within a city, and with other cities. Yet, Hillier et al. (2012) report some inconsistencies about the use of NAIN.

In SSx terminology global integration and choice measurements are carried out at city-wide spatial scales. Local integration and choice values are analysed by introducing metricized restriction radii (Hillier et al. 2010). High local integration values are associated with walkable areas that have dense and consolidated networks. High local choice values are associated with streets that serve to connect the neighbourhood-level areas to higher-hierarchy roads. Various integration values at increasing radii are argued to be empirically correlated with various types of movement patterns (Hillier et al. 1993; Hillier 1996; Hillier 2009; Penn 2003).

\section{Methodology}

\section{Case Study Areas}

Our case study cities are in Guatemala, Central America: Guatemala City (GC) and Quetzaltenango (QT). As in other countries in Latin America, the country has a colonial heritage in planning tradition (Ford 1996; Griffin and Ford 1980). This is reflected in historic gridiron networks and common Global South problems (Pacione 2005; Glebbeek and Koonings 2015) such as: heterogeneous and fragmented urban development, deteriorated historic cores, top-down, but weak planning practice and congestionrelated problems due to the uneven and unplanned horizontal expansion and centralized economic land uses. Both cities have expanded from an historic core, starting with planned expansions, and then moved towards unplanned peripheral developments following the main infrastructure (see Fig. 1). The first planned expansions are associated with current location of the core-business district (CBD). However, they differ significantly in size and stage of urban development, reflected in different streets configurations and ongoing economic dynamics. These differences made these cities adequate to the test applicability of our approach in different urban setups.

GC is the country's capital located in the central region. It accommodates around $26 \%$ of the country's population. It extends over $996 \mathrm{~km}^{2}$ within the municipal administrative boundary, excluding the conurbation areas in contiguous municipalities. Horizontal expansion is mainly shaped by topographic conditions. A segment of a non-finished peripheral ring connects the foundational core with the west 


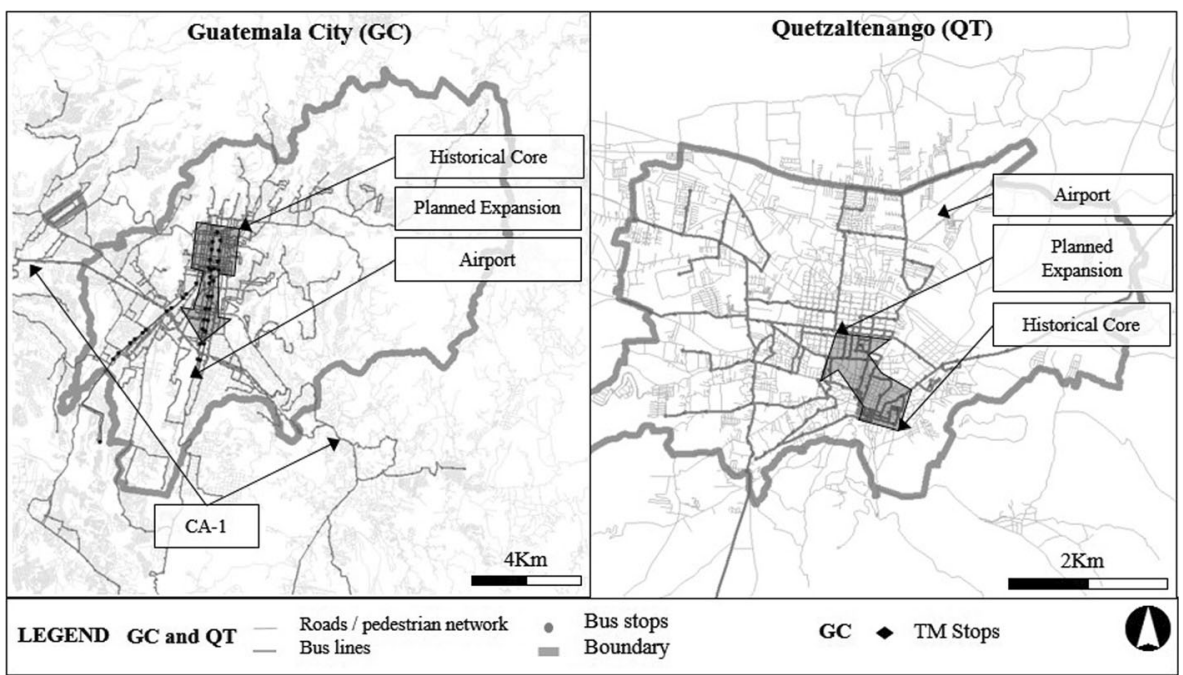

Fig. 1 Case study areas showing: Administrative boundaries, road and public transport network

and south-west areas. Current expansion mostly occurs in the south-eastern, southwestern and western areas, outside the administrative boundary. These areas are nurtured by the main infrastructure and the intra-regional CA-1 road.

QT is the second most important city, located in the west of the country. It accommodates around $5 \%$ of the population in a minor extension of $120 \mathrm{~km}^{2}$. Location of important infrastructure such as the airport, a peripheral road segment combined with topographical conditions influence current expansions mostly towards the northern and north-western areas. Still, further than the foundational core and the first expansion, a slow infill process is observable in the rest of the urban area.

\section{Data Collection, VGI Data and Pre-Processing}

Table 1 describes the data per accessibility approach that were used for the analyses. Official data were collected during fieldwork in the period of August 2014 to March 2015. The main problems with the data obtained from official institutions were: lack of up-to-dateness and incomplete geographical coverage. Data sets in GC only cover the administrative boundary, even though the functional city extends beyond those. Data from the contiguous municipalities do not exist. Therefore, we implemented a tailored approach that included extracting and pre-processing various sources of VGI.

Topological inconsistencies were the main problem when using OSM network data (Cooper and Chiaradia 2015; Cooper 2014; Gil 2015). Pre-processing involved the following steps: filtering out all the roads where vehicles are not allowed following OSM tags convention, planarizing the networks, except at overpass locations, removing duplicated features and detecting and correcting unconnected road segments. We simplified the networks using the Douglas-Peucker algorithm with an offset tolerance of $3 \mathrm{~m}$ for the SSx analyses. Then the simplified networks were fragmented at each vertex. 


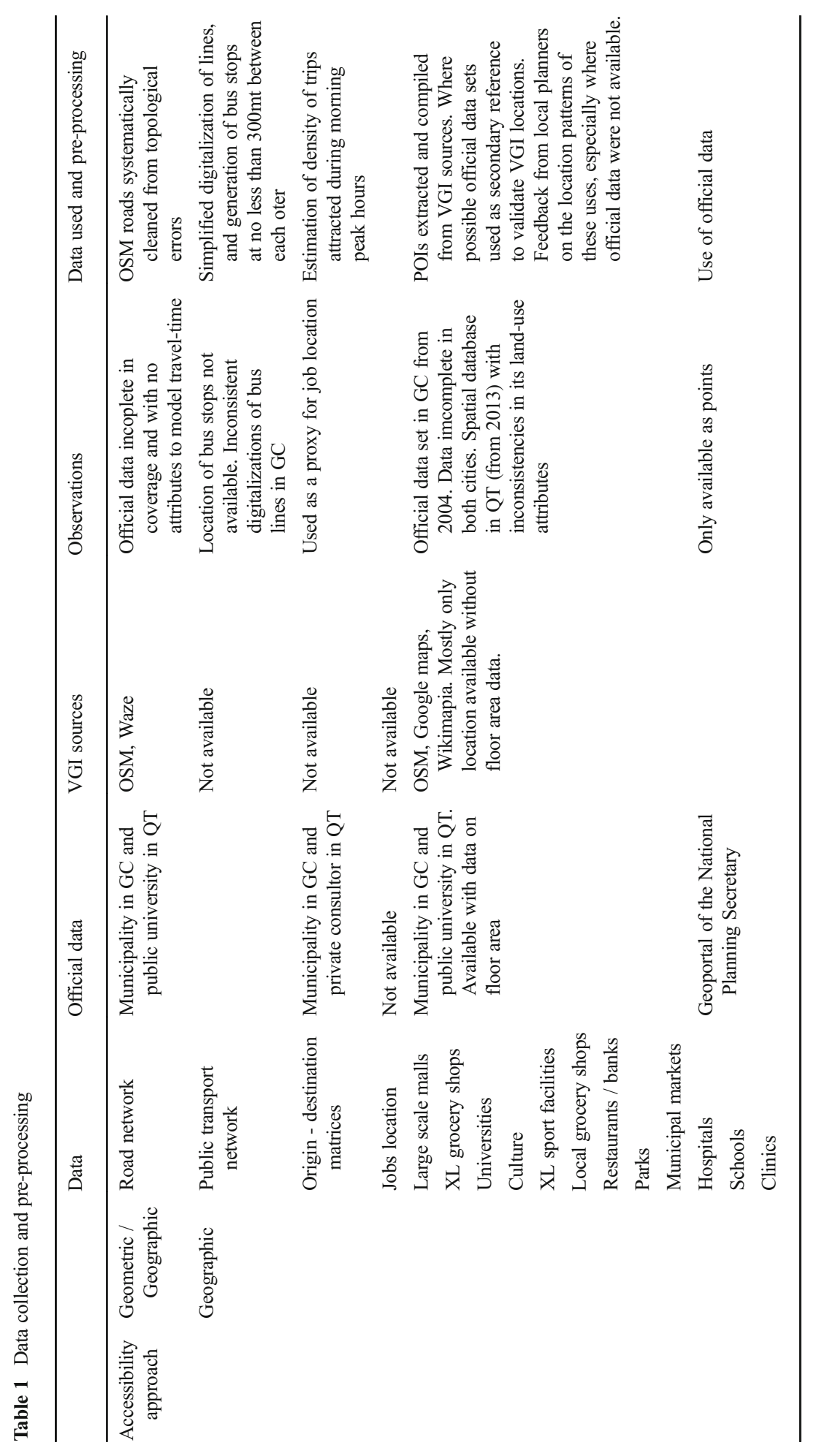


We built time-based network models for private-vehicle (PRIV) and public-transport mobility (PUB) for each city. First we examined for the correct categorical road classification based on OSM tags convention (e.g. motorway, primary, secondary, residential). A few important roads were found misclassified and were corrected. Speed limits were consulted with the respective authorities and added to the road segments according to their classification. Then we used Waze (Waze 2015) in GC and field observations in QT to calibrate final travel-times. The PUB models in both cities incorporate the roads as pedestrian networks. Travel-time calibration was carried out in consultation with local planners and experts.

In GC, origin-destination (OD) matrices per mode of transport (PRIV and PUB) during peak hours (6:00-9:00 am) were available for the year 2005. Each matrix contains 173 rows and columns, corresponding to 173 Traffic Analysis Zones (TAZ). Even though trip volumes in these data are outdated, we assumed they reflect overall mobility patterns that have not changed significantly. It means that the major attractors of morning trips, central locations of jobs and commerce, are the same up to date. In QT, OD data are more recent (2014) but drastically less detailed as each TAZ represents one of the 12 postal zones. Only the total number of trips attracted and generated during peak hours is available. Overall, the use of OD data was an alternative solution to overcome unavailable data on job locations. The total number of trips attracted was normalised per TAZ area and used as a proxy variable to indicate the density of job opportunities.

Table 1 shows that VGI was the main data source to compensate incomplete landuse data, extracted as points-of-interest (POIs). Still, exhaustive pre-processing was needed to use these data. In OSM most of the land uses were found as POIs, but in some cases they are digitised as polygons, or both. POIs of restaurants or banks within a large-scale mall (as POI or polygon) were simultaneously digitized, so they had to be detected and removed to avoid double counting. OSM conventions are not used consistently and the completeness of the data sets relies on the active contributors. Thus, other sources (GoogleMaps and Wikimapia) were used to cross-check and complete each land-use. Official land-use data was used mostly as a secondary reference. The final compiled datasets with POI locations were discussed with local planners to validate their use.

\section{Implementing Accessibility Analyses}

Figure 2 shows our methodological framework to map urban accessibility using a geographical and a geometrical approach. At a first glance, differences in data requirements are noticeable in each approach. In the geographical approach we started with the analysis of access to different land uses, by grouping them based on two sets of variables: macro- and micro-location. Macro-location addresses all those variables where, due to their characteristics (relevance and scale), people are more willing to overcome impedance. Micro-location addresses all locations with characteristics related to neighbourhood scale; thus, people are relatively less willing to overcome impedance. During the fieldwork we validated the relevance of these variables by means of a workshop with local experts in planning and real estate markets. Participants also ranked the variables on the basis of the relative importance of each variable to the local land market. 

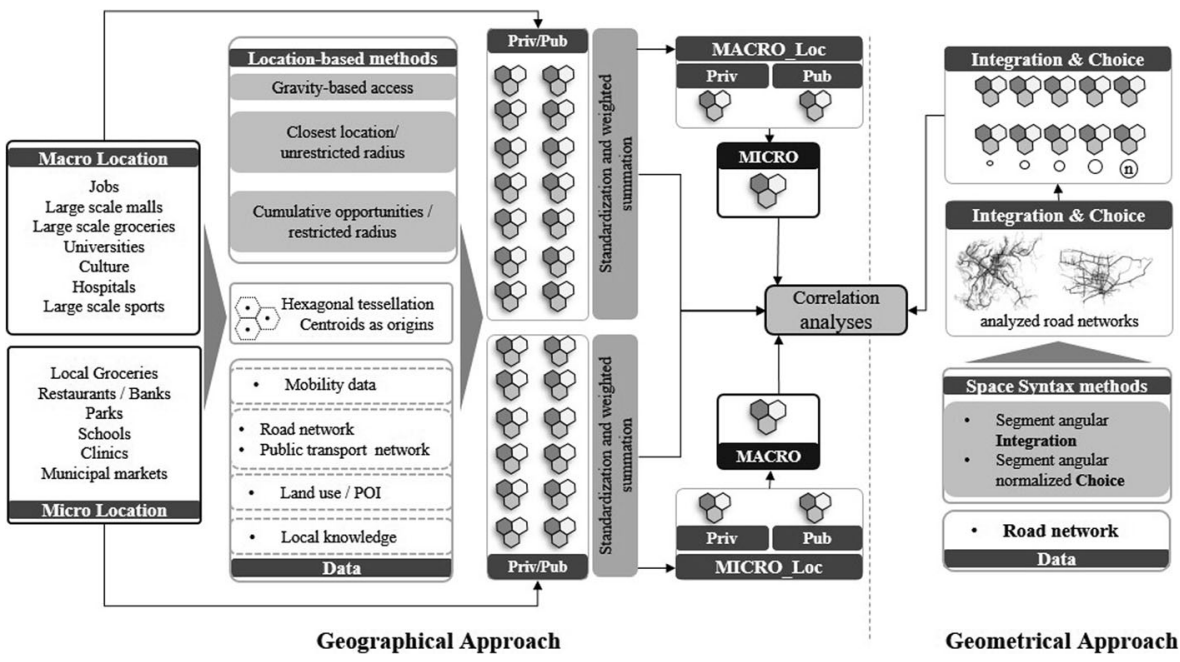

Fig. 2 Methodological framework

Geometrical Approach

We generated a hexagonal tessellation for each study area. A hexagonal shape was chosen for the following advantages: (1) spatial sampling performing slightly better when using geostatistical techniques compared to a grid sampling (Birch et al. 2007; Condat et al. 2005), if the analyses were to be used to investigate their relation with other socio-economic dynamics; (2) more symmetric nearest neighbourhoods, avoiding the ambiguities of a rectangular grid (Birch et al. 2007); (3) better visualization (Birch et al. 2007; Burdziej 2012). The size of the cells have a circumscribed diameter of $300 \mathrm{~m}$, equivalent to a distance of three blocks in the study areas. The size provides a reasonable resolution at a moderate computational demand. Cells that were not overlaying any roads were removed. The remaining hexagonal centroids were used as origins for the analyses.

We used the location-based methods described in section 2.1. The GC OD matrices were used to estimate the decay parameters $\boldsymbol{\alpha}$ and $\boldsymbol{\beta}$, following a probabilistic approach (Ingram 1971). Population-weighted centroids were used during this process to avoid aggregation biases (Hewko et al. 2002). Using ordinary least squares, decay functions per mode of transport were fixed as: $\boldsymbol{\alpha}=1.2$ and $\boldsymbol{\beta}=0.052$ for PRIV; $\boldsymbol{\alpha}=1.3$ and $\boldsymbol{\beta}=0.002$ for PUB. These values were used with eq. 2 and the density of trips attracted as " $M$ " to map access to jobs in both cities. The rest of the macro-variables were analysed using the shortest travel-time to reach a facility. All the micro-location variables were analysed using a cumulative-opportunity method, restricted to $10 \mathrm{~min}$ for both mobility modes.

Then we produced integrated maps per mobility mode (PRIV and PUB), at macro and micro level. First we standardized the results to a ' 0 ' - ' 1 ' scale using the non-linear standardization methods (Nyerges and Jankowski 2009). Then the results were combined per mode of transport using weighted summation. The ranking by experts was then used to estimate the normalized weights using the rank sum method (Malczewski 1999). Finally, the PRIV and $P U B$ results were also combined using the same procedure. The weights were equal to the percentage of trips generated per mode of transport 
from each TAZ. This step made the whole analysis sensitive to types of users and the accessibility per modality that benefits each location the most.

In the geometrical approach, we used SAA to analyse integration, choice, and their normalised value at different spatial radii. We started with a minimum radius equal to the average neighbourhood size $(0.8 \mathrm{~km})$. Then we expanded it to $1.5 \mathrm{~km}$ and $2.5 \mathrm{~km}$. From there we produced analyses by increasing the radius by $2.5 \mathrm{~km}$ up to the longest radius that would still produce information visually different than a global metric (7.5 $\mathrm{km}$ in GC and $5 \mathrm{~km}$ in QT). We aggregated the results, originally stored per road segment, to the hexagonal cells. Thus, each cell contains the average integration and the maximum choice values. Finally, we produced correlation matrices comparing the results from both approaches at $p<0.01$.

\section{Results and Discussion}

\section{Geographic-Accessibility}

Figures 3 and 4 show accessibility results per variable and per mode of transport for both cities. In GC, job access highly benefits the core area and rapidly decreases outwards. Access to large scale (XL) malls and grocery shops mostly benefit a corridor area from north-west to south-east. Access to universities and hospitals tend to benefit the most central east-western areas. Access to culture and XL sport-related facilities (e.g. stadiums) outlines a north-south corridor. At the micro-location, low and mediumlow access predominates in the maps. Still, the core corridor simultaneously benefits from access to multiple locations of grocery shops, banks and restaurants, schools, clinics and municipal markets. Access to parks points out an urban area in the northwest. Here, various small parks are found within residential neighbourhoods.

The core area shows a relatively low-medium parks access. However, important public and larger open spaces are located in this corridor; especially in the historic CBD. This outlines the limitation of not including the size of each facility in the analysis. Overall, public transport availability and travel-times strongly constrain accessibility at macro and micro-location scale. Repeatedly, the north-eastern areas lack good accessibility.

In QT, private access to jobs, cultural facilities and XL sports favours the most central and historic areas. High access to XL malls and XL grocery shops reflects an emerging commercial pole located in the north-eastern area. Highest access to universities and hospitals are less associated with high access to other variables. Overall, public transport restricts high accessibility to different variables, mostly to core areas. Public transport availability and quality highly restricts accessibility. Mostly, central and historic areas benefit from high access to various land uses. The central area still benefits from medium high access to XL malls and grocery shops. Also, it benefits the most from high access to all the micro-location variables using both mobility modes.

Figures 5 and 6 show integrated macro- and micro-location accessibility maps for PRIV (a), PUB (b) and both modes combined (c) for each city. Standardized "s" colour ranges from Figs. 3 and 4 are applicable to these figures. Accessibility scores range from 0 (red) to 100 (green). Road segments scoring high SSx NACH values, classified 


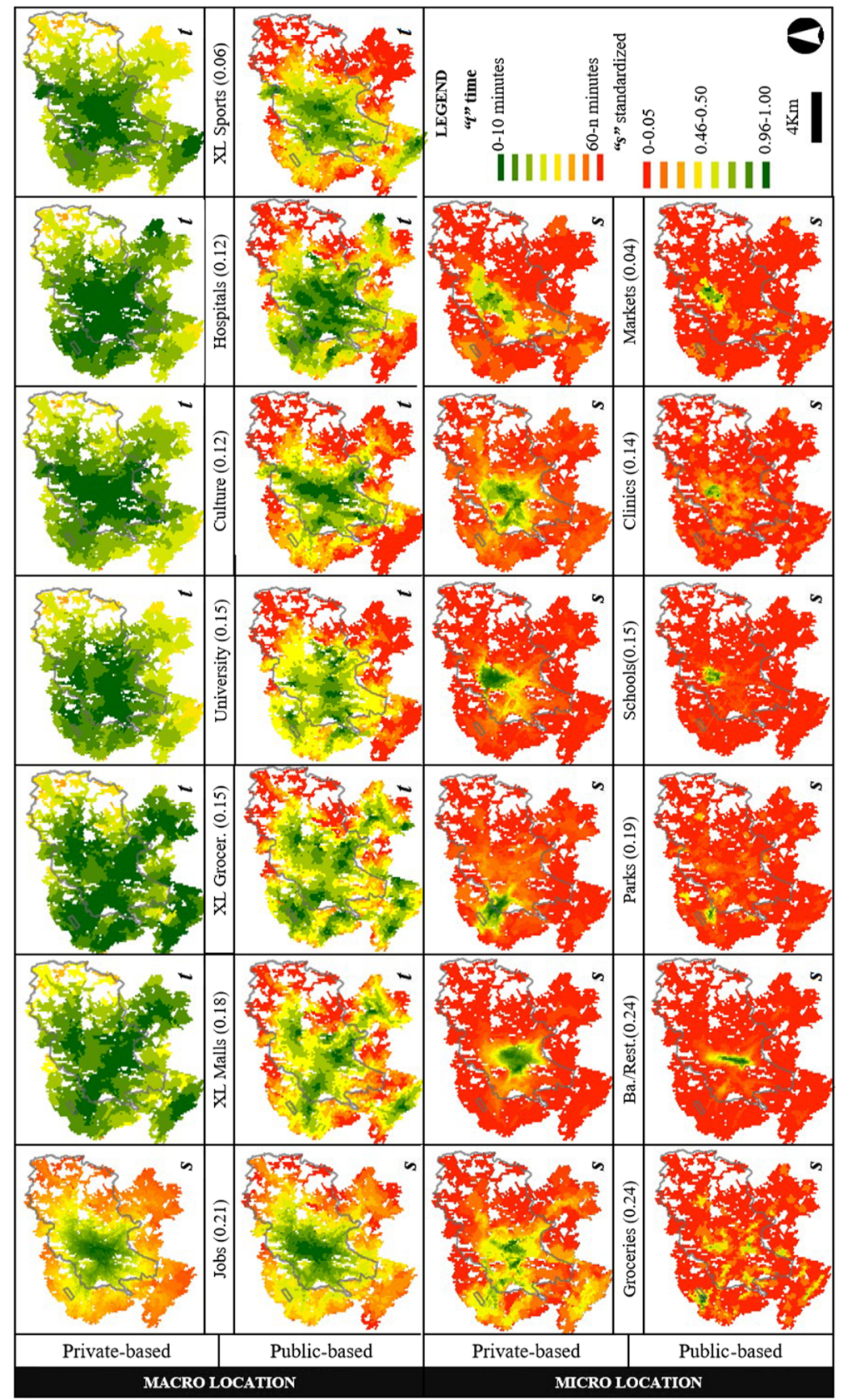

Fig. 3 Accessibility per variable per mode of transport in Guatemala City

in two, are overlaid on the access maps showing the combined scores (c). Discussion of this layer is provided in next sections.

In GC the core corridor benefits the most from PRIV and PUB access given the current mobility infrastructure to access macro-location variables (maps "a" and "b"). 


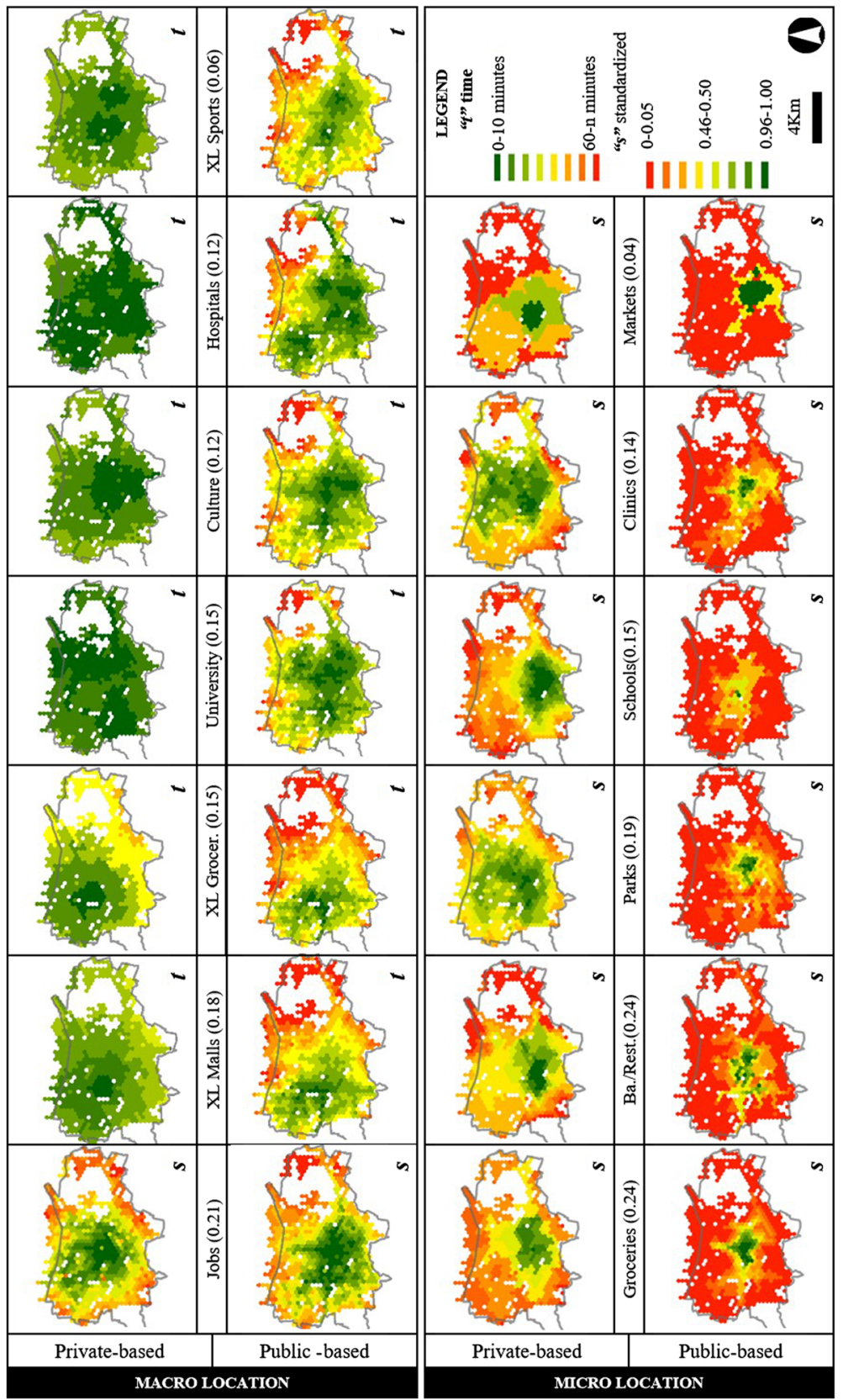

Fig. 4 Accessibility per variable per mode of transport in Quetzaltenango

The combined effect of macro and micro-location accessibility, maps "c", is mostly influenced by PUB accessibility. That is because the average percentage of public transport users per TAZ is higher $(0.76)$ than private vehicle ones $(0.24)$. Highest macro accessibility (scores 0.91-1) outlines three important areas in map (c): the current CBD 


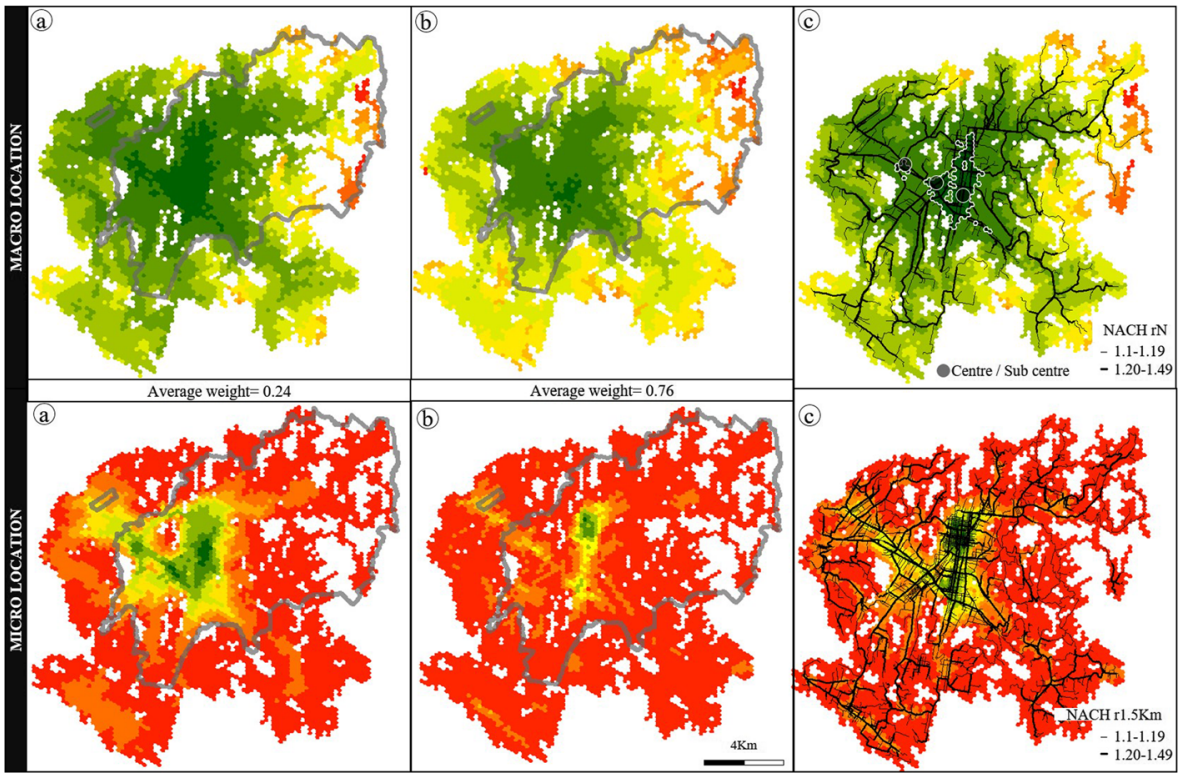

Fig. 5 Integrated macro and micro-location accessibility for Guatemala City (same legend as in figs. 3 and 4)

and two inter-connected sub-centres aligned along the CA-1. These cores match the locations perceived by local experts as important economic centres and important job sources. Contrastingly, current policies are mostly focused on centrality at the northsouth core corridor. However, an opportunity to strengthen an existing polycentric structure extends towards the north-west. Micro-location emphasises the historic centre, core corridor, and quickly decreases towards the other sub-centres with medium scores. Medium-low scores $(0.3-0.4)$ point out areas that benefit from minor concentrations to a combination of various micro variables.

In QT the modal split is equal for the whole area (50\%-50\%). The highest combined macro accessibility benefits central areas and extends towards the emerging commercial pole. Accessibility slowly decreases towards the periphery. The east periphery has

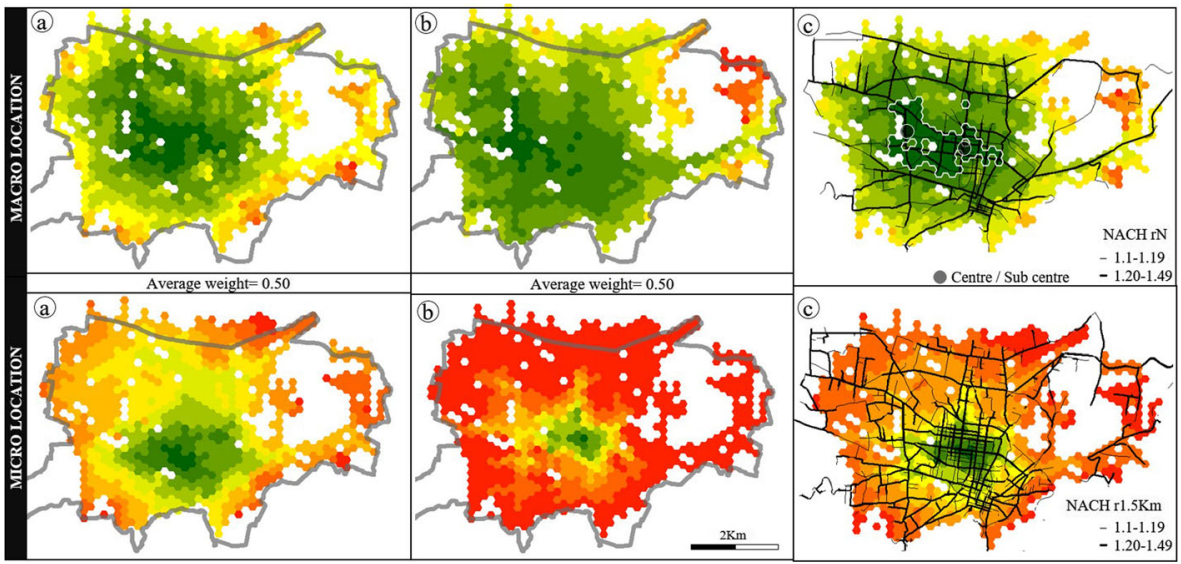

Fig. 6 Integrated macro and micro-location accessibility for Quetzaltenango (same legend as in figs. 3 and 4) 
the lowest macro accessibility. Micro-location benefits core areas the most, including the historic centre. Accessibility quickly decreases towards the periphery. Unlike GT, the emerging pole benefits the most only from high macro accessibility.

\section{Geometric-Accessibility}

Figure 7 shows the SSx results of integration and $\mathrm{NACH}$ at selected radii. In GC, the core areas benefit the most from geometric accessibility at various spatial scales. Neighbourhood scale integration $(\mathrm{r} 0.8 \mathrm{~km})$ outlines urban areas with compact grids (small blocks). These areas correspond to some of the old planned neighbourhoods at the core areas and the core settlements of the peripheral municipalities. These areas have the highest potential for pedestrian movements. High integration (1700 ) at r5 km highlights the core corridor and extends towards the southern part following important

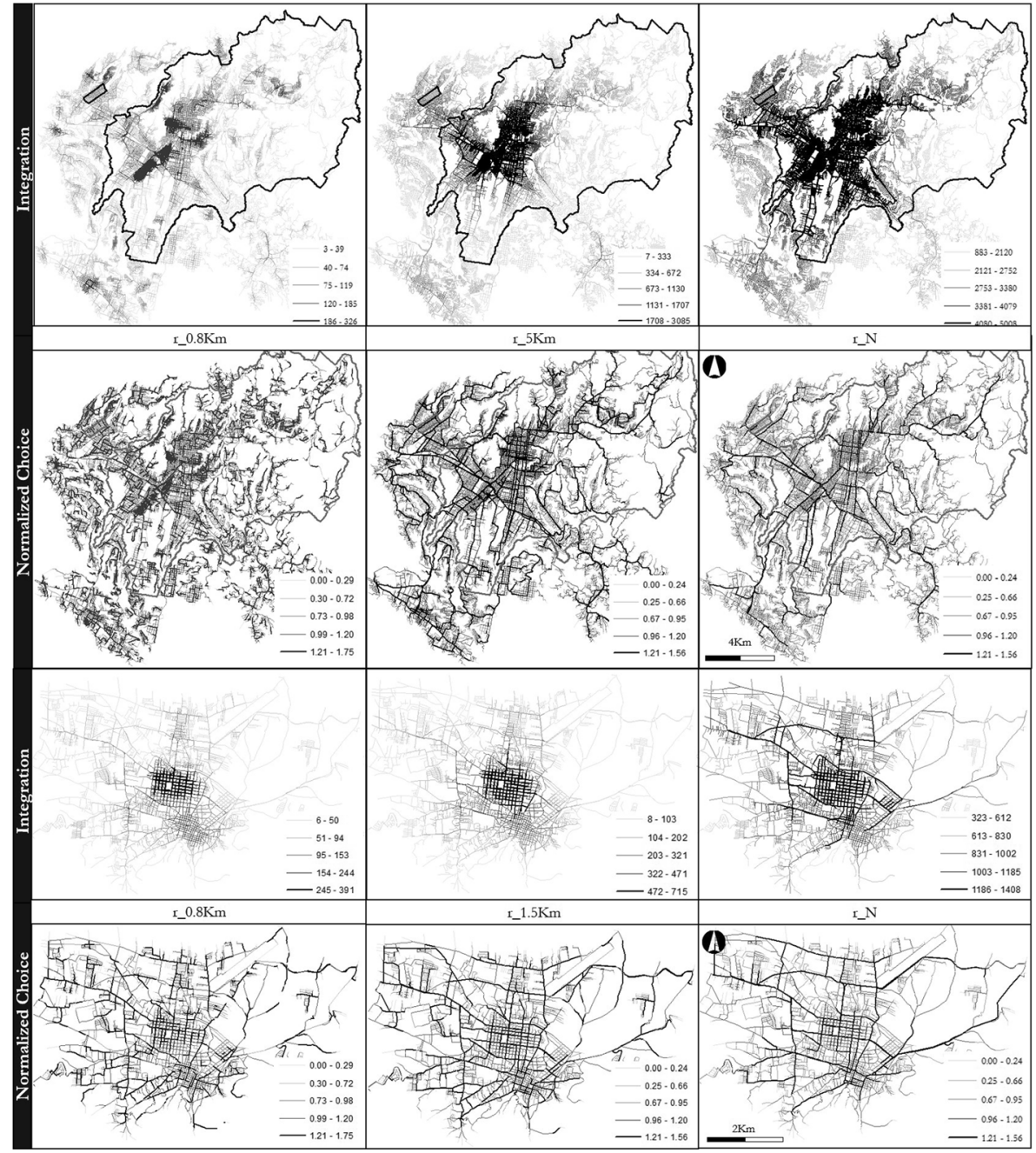

Fig. 7 SSx results for Guatemala City and Quetzaltenango 
roads. Eastern, southern and western peripheral areas account for medium integration (600-100), while some areas in between these and the core account for lower integration values. Overall, these patterns already provide visual insights on the important association between integration and the agglomeration of economic and service activities in the core areas versus the peripheral areas, as outlined by geographic access. This predominance is reflected in the disposition of city structuring roads when observing $\mathrm{NACH}$ analyses $(\mathrm{r} 5 \mathrm{~km}$ and $\mathrm{rN})$. The core corridor is the only area framed and traversed by these important roads, while towards the periphery the urban patches are connected to this framed core by treetype configurations. The peripheral roads play an important role structuring the western area, producing an important intersection where one of the sub-centres is outlined via macro accessibility.

In QT the central area is highly integrated at different spatial scales, more than the historic centre, which is associated with the high centrality of such an area as visualized via geographic access. High integration at $\mathrm{rN}$ outlines areas towards the north-west, matching the location of the emerging commercial pole. Contrastingly, other peripheral areas are poorly integrated at different spatial scales, denoting less consolidated urbanization. High NACH values at the lowest radius outline service roads at neighbourhood scale. Similarly to GC, both the central area and the historic centre seem to be well connected by means of city structuring roads outlined by NACH analyses (r $1.5 \mathrm{~km}$ and $\mathrm{rN}$ ). Those connect to other important roads: a periphery eastwest road on the north (intersecting with the emergent pole) and a major south-north road on the west side.

During the analyses, integration and NAIN were both explored. However, we observed inconsistencies of the normalized measurement at low radii, as reported in Hillier et al. (2012). In GC, analyses at $\mathrm{r} 0.8 \mathrm{~km}$ and $1.5 \mathrm{~km}$ were highlighting isolated segments in peripheral areas. In QT, the same inconsistencies were observed with analyses at $\mathrm{r} 0.8 \mathrm{~km}, 1.5 \mathrm{~km}, 2.5 \mathrm{~km}$ and even $5 \mathrm{~km}$. This was expected as the urban layout in QT is less consolidated compared to GT.

\section{Associations between the Geographical and the Geometrical Approach}

Figures 8 and 9 show the results of Pearson correlation matrices $(p<0.01)$ between geographical (location-based) and geometrical (SSx) accessibility, per mobility mode for each city. Square size and colour range, from small to large and from white to black, indicate correlation strength. The matrices reveal the associations of the various accessibility measures within and between approaches. We confirm a positive correlation between geometric and geographic accessibility to various facilities and aggregated macro- and micro-location accessibility. The results do not indicate a directional causality. However, high geographic accessibility is the result of location and concentration of various land uses. Various land uses are more prone to change or relocate over time, compared to changes in the urban layout. Thus, we could think of the geographic accessibility as a result of a cumulative process of land-use location influenced by seeking optimal geometric location. We first discuss the correlations between the various location-based analyses and then the correlations between locationbased measurements and SSx. 


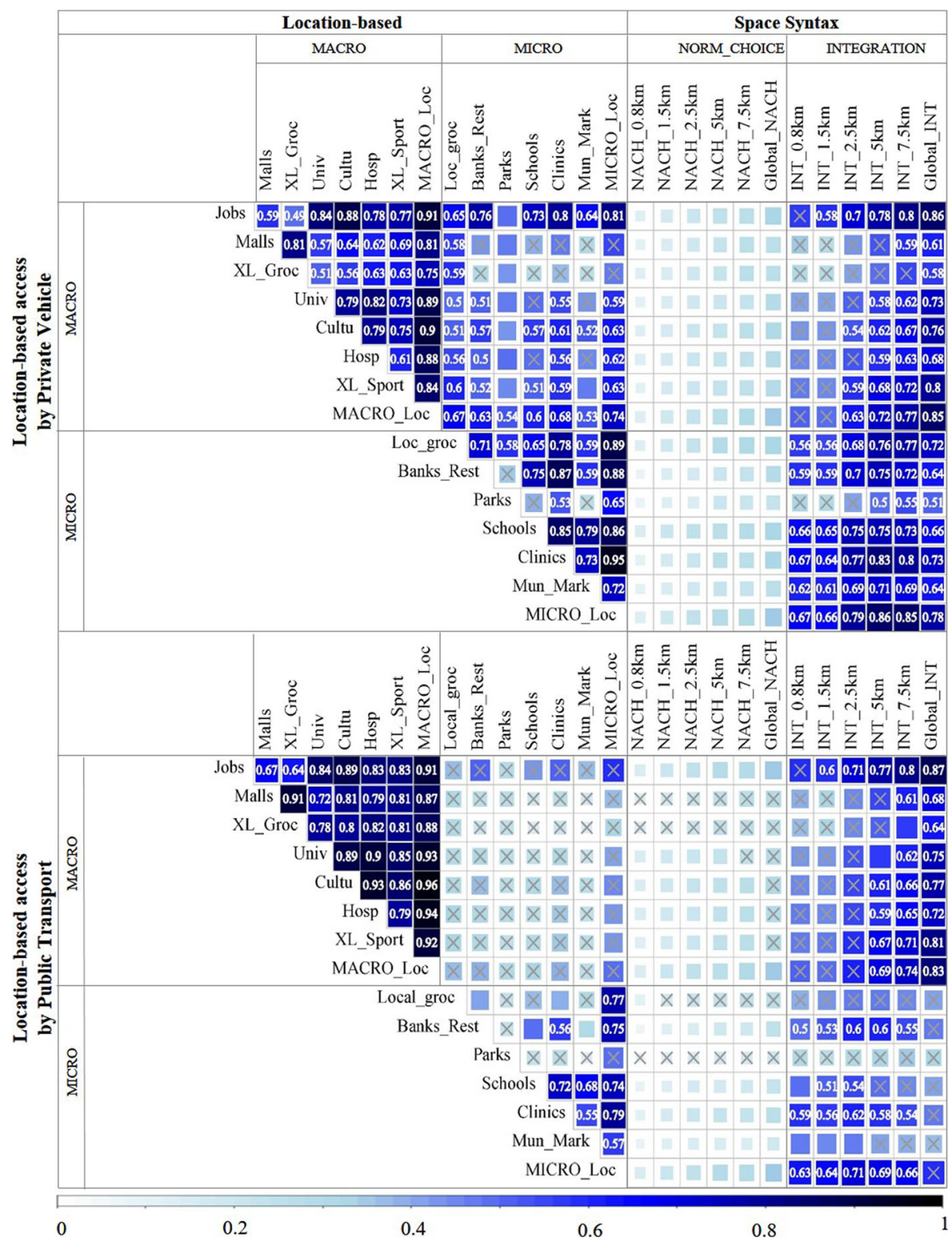

Fig. 8 Pearson correlation matrix $(\mathrm{p}<0.01)$ between geographical (location-based) and geometrical (SSx) accessibility in Guatemala City. Insignificant correlations are cross marked. Correlation values are indicated only for correlations $>0.50$.

Figure 8 shows that the distribution of PRIV accessibility in GC to various macro-variables is slightly less similar compared to PUB. While PRIV mobility infrastructure is more evenly distributed, varying locations of macro-variables produce more irregular patterns. It reflects that some areas do not benefit simultaneously of the same access levels to all macro-variables. In turn, the PUB infrastructure restricts homogeneously higher access to macro-variables only to 


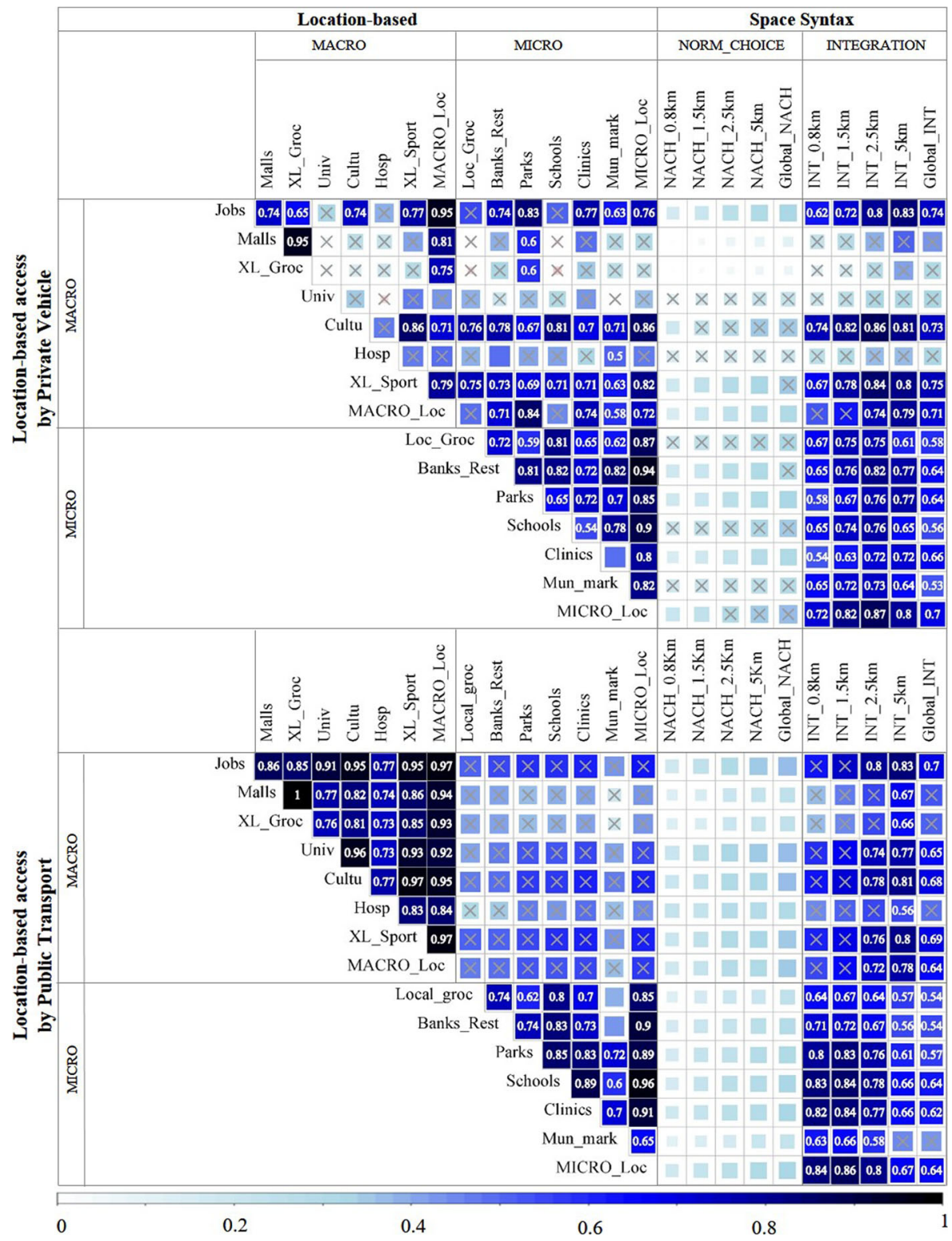

Fig. 9 Pearson correlation matrix $(\mathrm{p}<0.01)$ between geographical (location-based) and geometrical (SSx) accessibility in Quetzaltenango. Insignificant correlations are cross marked. Correlation values are indicated only for correlations $>0.50$.

the core areas. The remaining areas have simultaneously poorer access to multiple macro-variables. Higher and significant correlations between macro-variables and micro-variables in the upper matrix outline that only PRIV mobility offers simultaneous access to macro- and micro-variables, unlike PUB.

Figure 9 shows that in QT PRIV accessibility patterns to malls, XL-grocery shops, hospitals and universities differ highly from accessibility to various other land uses. 
Partially, this is explained as the first two are located in the emerging commercial-pole. In turn, the PUB infrastructure simultaneously benefits with high accessibility to the same areas, a reduced portion of the city. The rest simultaneously benefits from medium or poor access to various macro-variables. Access distributions to microvariables are significantly similar both for PRIV and PUB. Unlike the location of macro-variables, these are concentrated in the same areas. Similar to GC, significant and insignificant correlations between the macro with the micro-variables show that PRIV mobility means higher access to both groups of variables than PUB mobility.

Potential PRIV access to jobs strongly correlates with various location-based metrics in both cities. The cumulative access to areas that attract more trips during morning peak hours works as a latent variable showing areas favoured by good access to various commercial and service type of land uses (job sources). As was expected, it emphasizes the important role of such a metric to understand the distribution of economic opportunities, as well as its potential to visualize aggregated geographic access via a robust location-based metric using only trip data.

The highest correlations between location-based measurements (PRIV, PUB) and $\mathrm{NACH}$ were found at $\mathrm{rN}$ in both cities. Although not all of them are significant at $p<0.01$, they are at $p<0.05$. In QT correlations between PRIV location-based accessibility to malls, XL-grocery shops and NACHrN are insignificant in both cases. Even though correlation levels are low, these modestly provide evidence of location preference of profitable or service land uses. It shows an association between route choices as a function of travel time with a cognitive criteria of least angular deviation. By looking at maps c in Figs. 5 and 6, we could suggest that $\mathrm{NACH}$ metrics provide relevant information that is complementary to location-based metrics by outlining those structuring roads from where geographic access distributes across a city. We observe that the distribution of location-based accessibility closely follows the arrangement of the important roads. Furthermore, this confirms the preference of the various land uses for roads where higher flows of people are expected.

Macro variables consistently show the highest correlation with high radii integration, $\mathrm{rN}$ in GC and mostly with $\mathrm{r} 5 \mathrm{~km}$ in QT. This difference shows the effect of the different territorial extensions and stages of urban development. In QT, high $\mathrm{rN}$ integration is associated with a higher concentration of economic activities, or at least where there is potential for those. The emergence of the west economic pole responds to this potential. However, location-based access to most of macro-variables tends to outline more the central areas. Therefore, high SSx integration at $\mathrm{r} 5 \mathrm{~km}$ captures better the current location of most of these uses. Furthermore, this also explains the disassociation between PRIV location-based accessibility to malls and XL-grocery shops, and integration values.

In both cities micro-location accessibility shows the highest correlation with integration at lower radii. We found that lower radii reflect restricted time-based mobility in two ways: the 10-min threshold imposed in the analysis and mobility restrictions imposed by the current public transport infrastructure. In GC PRIV micro-access shows the highest correlation with integration-r5 $\mathrm{km}$ and PUB with $\mathrm{r} 2.5 \mathrm{~km}$. In QT PRIV micro-access shows the highest correlation with integration-r2.5 km and PUB at $\mathrm{r} 1.5 \mathrm{~km}$. These differences also show that micro-location land-uses in QT are spatially distributed and more accessible to pedestrian movements, compared to GC.

The correlations provide insights about how integration at different spatial scales (radii) is associated with access to various land uses. SSx analyses the geometric access 
as an aggregated resource, assumed to be preferred by simultaneous location and concentration of the various land uses. Aggregated location-based variables (Macro_loc and Micro_loc) attempt to reflect this dynamic. In our case, aggregated Macro_loc and Micro_loc address an expert-based ponderation of each land use regarding the local land market. As expected, both aggregated variables are associated the most with $\mathrm{NACH}$ values at $\mathrm{rN}$ in both cities, no matter the transport-mode. Correlation between Macro_loc and integration in GC peaks at $\mathrm{rN}$ and at $\mathrm{r} 5 \mathrm{~km}$ in QT for both transport modes. The relative importance of access to jobs becomes evident in the case of QT when addressing PUB access. In GC Micro_loc is associated most with integration at r5 km-r7.5 km for PRIV access and r2.5 km-r5 km for PUB access. In QT Micro_loc is associated most with integration at $\mathrm{r} 2.5 \mathrm{~km}$ and $\mathrm{r} 1.5 \mathrm{~km}$ correspondingly. The divergence per mode of transport in both cities emphasizes the mobility restrictions posed by PUB mobility using the same 10-min travel-time, but more evident for the case of QT.

Location-based methods were adapted to consider the accessibility components as long as data allowed it. The results are rich in information about accessibility to various land uses: indeed, 32 maps per city. In turn, the geometrical approach considered only two measurements (integration and choice) at different analysis radii: 12 maps per city. SSx outputs are intuitive in terms of visualizing accessible locations, where we can assume that profitable uses or service facilities are present. Differences in data requirements and pre-processing give significant advantage to the geometrical approach. However, previous training on SSx and local knowledge is required for adequate interpretation. Besides, the applicability of the SSx might be limited in cases such as QT, where ongoing development is sparse. Furthermore, VGI was applicable in both approaches in this research. After exhaustive pre-processing these data became indispensable to implement the geographic approach. In turn, data for a geometric approach could be manually or digitally derived from other sources such as satellite imagery.

\section{Conclusions}

Urban accessibility was successfully mapped using a geographical and a geometrical approach and the results were compared using correlations. We found consistent correlations between accessibility measurements from both approaches, which allows us to confirm our initial hypothesis. The results do not explain directionality of the causal relation between geometrical and geographic accessibility, but it is logical to think that in Global South cities with weak land-use policies, geometric accessibility influences a location process where various land uses seek to benefit from reachable locations (integration) and exposure to movement (choice).

Our research does not solve the analytical dual approach problem outlined in Batty (2013). However, the results establish an empirical connection between the morphological-geometric properties of an urban area and the distribution of timebased geographic accessibility. The quantitative relations provide additional knowledge on the interpretability and limitations of the information that is produced in Space Syntax using little-data. Our results are placed in the context of studies such as Kim and Sohn (2002) and van Nes et al. (2011), where positive associations are claimed between 
SSx metrics with building density and location of various land uses, which is observed in the present work, and is in line with Space Syntax capacity to aid in predicting flows of people (Hajrasouliha and Yin 2015).

The consistency of the correlations between geographic and geometrical measurements simultaneously validates the use of VGI, and the applicability of Space Syntax in these two cities. Location-based analyses using VGI data produced plausible results that outlines how access to various land uses is distributed across each city. In turn, Space Syntax analyses applied to different urban contexts produced information that is statistically comparable with the results of a location-based approach. Based on the correlation results we claim that a geometrical approach using SSx delivers plausible information from where inferences could be made about geographic accessibility. Space Syntax turns out to be a more data/time efficient approach.

We found some limitations when applying Space Syntax in less consolidated urban areas, besides the problems with the normalised measurements reported in Hillier et al. (2012). Global integration measurements might not reflect the ongoing land-use processes in less-consolidated areas consistently. We cannot conclude from the results which spatial radii best describe geographic access, as these differed per city. This is one important drawback of SSx analysis, as there is not enough evidence to suggest which spatial radii correlate better with what in different cities. This observation could also apply to the MCA method (Porta et al. 2012). Further research could address these observations by replicating the research in a larger set of case studies and expanding the comparisons by including network centrality metrics from other methods, such as the MCA.

The methodologies and results are important for the planning practice. In Guatemala the results provide important information for transport and land-use planning, which was not available before. Mobility and land-use projects could be assessed using the methodologies presented here. We suggest that our methodological framework, including the use of VGI, is replicable to other cities in the region and Global South, where data are scarce but information is highly relevant for planning tasks. Space Syntax would be a valid approach for planners and researchers in areas where not even VGI is available, for example, by analysing the attractiveness of places, the city structure, or geometric accessibility impacts of changes in street configuration. Although the outputs of both approaches are statistically comparable, rather than replacing location-based methods, Space Syntax offers an important complementary measure to geographical accessibility. We describe Space Syntax as an accessibility tool able to support early-stage planning processes when limited data are available. Trained interpretations enriched with local knowledge could provide first-level insights on concentrations of profitable and public services land use, and the distribution of access to those. Such interpretations could be further examined in detail for areas of interest using location-based methods, potentially supported by VGI. Finally, accessibility analysis could benefit from addressing time, land-use and geometric aspects to improve the understanding in other fields such as human geography and urban economy in cities in the Global South. 
Acknowledgements We would like to thank the collaboration by local experts from different offices: Guatemala City "Direccion de Mobilidad Urbana", "Secretaría de Planificación y Programación de la Presidencia", "Centro Universitario de Occidente", "Inspecciones Globales" and "Fab Lab". The work reported in this research is funded by NUFFIC through the NICHE Project.

Open Access This article is distributed under the terms of the Creative Commons Attribution 4.0 International License (http://creativecommons.org/licenses/by/4.0/), which permits unrestricted use, distribution, and reproduction in any medium, provided you give appropriate credit to the original author(s) and the source, provide a link to the Creative Commons license, and indicate if changes were made.

\section{References}

Ahlström, A., Pilesjö, P., \& Lindberg, J. (2011). Improved accessibility modeling and its relation to poverty A case study in Southern Sri Lanka. Habitat International, 35(2), 316-326.

Albacete, X., Olaru, D., Paül, V., \& Biermann, S. (2015). Measuring the accessibility of public transport: A critical comparison between methods in Helsinki. Applied Spatial Analysis and Policy, 1-28. https://doi. org/10.1007/s12061-015-9177-8.

Arsanjani, J. J., Zipf, A., Mooney, P., \& Helbich, M. (2015). An introduction to OpenStreetMap in geographic information science: Experiences, research, and applications. In OpenStreetMap in GIScience (pp. 1-15). Springer International Publishing.

Bafna, S. (2003). Space syntax: A brief introduction to its logic and analytical techniques. Environment and Behavior, 35(1), 17-29.

Batty, M. (2004). A new theory of space syntax. London: Centre of Advanced Spatial Analysis UCL.

Batty, M. (2009). Accessibility: In search of a unified theory. Environment and Planning. B, Planning \& Design, 36(2), 191-194.

Batty, M. (2013). The new science of cities. Cambridge: Mit Press.

Birch, C. P., Oom, S. P., \& Beecham, J. A. (2007). Rectangular and hexagonal grids used for observation, experiment and simulation in ecology. Ecological Modelling, 206(3), 347-359.

Burdziej, J. (2012). A web-based spatial decision support system for accessibility analysis - concepts and methods. Applied Geomatics, 4(2), 75-84.

Condat, L., Van De Ville, D., \& Blu, T. (2005). Hexagonal versus orthogonal lattices: A new comparison using approximation theory. In Image Processing, 2005. ICIP 2005. IEEE International Conference (Vol. 3, pp. III-1116-1119). IEEE.

Cooper, C. (2014). Using OpenStreetMap in spatial network analysis. Cardiff University: sDNA.

Cooper, C., \& Chiaradia, A. (2015). sDNA: How and why we reinvented spatial network analysis for health, economics and active modes of transport. In: Malleson et al, N. (Ed.), GIS Research UK (GISRUK) 2015 Proceedings. https://figshare.com/articles/GIS_Research_UK_GISRUK_2015_Proceedings/1491375.

Curl, A., Nelson, J. D., \& Anable, J. (2011). Does accessibility planning address what matters? A review of current practice and practitioner perspectives. Research in Transportation Business \& Management, 2, 3-11.

Curtis, C., \& Scheurer, J. (2010). Planning for sustainable accessibility: Developing tools to aid discussion and decision-making. Progress in Planning, 74(2), 53-106.

Dalton, R. C. (2003). The secret is to follow your nose. Environment and Behavior, 35(1), 107-131. https://doi.org/10.1177/0013916502238867.

Ford, L. (1996). A new and improved model of Latin American City structure. Geographical Review, 86(3), $437-440$

Freeman, L. C. (1977). A set of measures of centrality based on betweenness. Sociometry, 40(1), 35-41.

Geurs, K. T., \& van Eck, R. (2001). Accessibility measures, review and applications (Vol. 4085050, RIVM rapport $=$ RIVM report). Bilthoven: Rijksinstituut voor Volksgezondheid en Milieu (RIVM).

Geurs, K., \& Van Wee, B. (2004). Accessibility evaluation of land-use and transport strategies: Review and research directions. Journal of Transport Geography, 12(2), 127-140.

Gil, J. (2015). Building a multimodal urban network model using OpenStreetMap data for the analysis of sustainable accessibility. In OpenStreetMap in GIScience (pp. 229-251). Springer International Publishing.

Glebbeek, M. L., \& Koonings, K. (2015). Between Morro and Asfalto. In Violence, insecurity and sociospatial segregation in Latin American cities. Habitat: International, 54(1),3-9. 
Griffin, E., \& Ford, L. (1980). A model of Latin American City structure. Geographical Review, 70(4), 397422. https://doi.org/10.2307/214076.

Hajrasouliha, A., \& Yin, L. (2015). The impact of street network connectivity on pedestrian volume. Urban Studies, 52(13), 2483-2497. https://doi.org/10.1177/0042098014544763.

Handy, S. L., \& Niemeier, D. A. (1997). Measuring accessibility : An exploration of issues and alternatives. Environment and Planning A, 29(7), 1175-1194.

Hansen, W. G. (1959). How accessibility shapes land use. Journal of the American Institute of Planners, 25(2), 73-76.

Hewko, J., Smoyer-Tomic, K. E., \& Hodgson, M. J. (2002). Measuring neighbourhood spatial accessibility to urban amenities: Does aggregation error matter? Environment and Planning A, 34(7), 1185-1206.

Hillier, B. (1996). Cities as movement economies. Urban Design International, 1(1), 41-60.

Hillier, B. (2009). Spatial sustainability in cities: Organic patterns and sustainable forms. Paper presented at the 7 th international space syntax symposium, Stockholm.

Hiller, B., \& Iida, S. (2005). Network and psychological effects in urban movement. In A. G. Cohn, \& D. M. Mark (Eds.), Proceedings of spatial information theory: International conference 2005 (pp. 468-476). Ellicottsville: COSIT.

Hillier, B., \& Penn, A. (2004). Rejoinder to Carlo Ratti. Environment and Planning. B, Planning \& Design, 31(4), 501-511.

Hillier, B., Leaman, A., Stansall, P., \& Bedford, M. (1976). Space syntax. Environment and Planning B, 3(2), $147-185$.

Hillier, B., Penn, A., Hanson, J., Grajewski, T., \& Xu, J. (1993). Natural movement-or, configuration and attraction in urban pedestrian movement. Environment and Planning B, 20(1), 29-66.

Hillier, B., Greene, M., \& Desyllas, J. (2000). Self-generated Neighbourhoods: The role of urban form in the consolidation of informal settlements. Urban Design International, 5(2), 61-96.

Hillier, B., Turner, A., Yang, T., \& Park, H.-T. (2010). Metric and topo-geometric properties of urban street networks: Some convergences, divergences and new results. Journal of Space Syntax Studies, 1(2), 258279.

Hillier, B., Yang, T., \& Turner, A. (2012). Normalising least angle choice in Depthmap-and how it opens up new perspectives on the global and local analysis of city space. Journal of Space Syntax, 3(2), 155-193.

Ingram, D. R. (1971). Concept of accessibility : A search for an operational form. Regional Studies, 5, $101-107$.

Jiang, B., \& Claramunt, C. (2002). Integration of space syntax into GIS: New perspectives for urban morphology. Transactions in GIS, 6(3), 295-309.

Karimi, K. (2012). A configurational approach to analytical urban design: 'space syntax'methodology. Urban Design International, 17(4), 297-318.

Kim, H.-K., \& Sohn, D. W. (2002). An analysis of the relationship between land use density of office buildings and urban street configuration: Case studies of two areas in Seoul by space syntax analysis. Cities, 19(6), 409-418.

Koenig, J.-G. (1980). Indicators of urban accessibility: Theory and application. Transportation, 9(2), 145-172.

Kourtit, K., Nijkamp, P., \& Partridge, M. D. (2015). Challenges of the new urban world. Applied Spatial Analysis and Policy, 8(3), 199-215. https://doi.org/10.1007/s12061-015-9155-1.

Law, S. (2017). Defining street-based local area and measuring its effect on house price using a hedonic price approach: The case study of metropolitan London. Cities, 60, Part A, 166-179.

Malczewski, J. (1999). GIS and multicriteria decision analysis. New York: Wiley.

Matthews, J., \& Turnbull, G. (2007). Neighborhood street layout and property value: The interaction of accessibility and land use mix. The Journal of Real Estate Finance and Economics, 35(2), 111-141.

Netzell, O. (2012). The effect of accessibility on retail rents: Testing integration value as a measure of geographic location. Journal of Property Research, 30(1), 1-23. https://doi.org/10.1080 /09599916.2012.713974.

Nyerges, T. L., \& Jankowski, P. (2009). Regional and urban GIS: A decision support approach. New York: Guilford Press.

Pacione, M. (2005). Urban geography : a global perspective (second edition ed.). London: Routledge.

Penn, A. (2003). Space syntax and spatial cognition or why the axial line? Environment and Behavior, 35(1), 30-65.

Porta, S., Crucitti, P., \& Latora, V. (2005). The network analysis of urban streets: A primal approach. Environment and Planning B, 33(5), 705-725.

Porta, S., Crucitti, P., \& Latora, V. (2006). The network analysis of urban streets: A dual approach. Physica A: Statistical Mechanics and its Applications, 369(2), 853-866.

Porta, S., Latora, V., \& Strano, E. (2010). Networks in urban design. Six years of research in multiple centrality assessment. In Network science (pp. 107-129). Springer London. 
Porta, S., Latora, V., Wang, F., Rueda, S., Strano, E., Scellato, S., et al. (2012). Street centrality and the location of economic activities in Barcelona. Urban Studies, 49(7), 1471-1488. https://doi.org/10.1177 /0042098011422570.

Ratti, C. (2004). Urban texture and space syntax: Some inconsistencies. Environment and Planning. B, Planning \& Design, 31(4), 487-499.

Rietveld, P. (2015). Flying carpets and urban development trajectories. Applied Spatial Analysis and Policy, 8(3), 217-230. https://doi.org/10.1007/s12061-015-9142-6.

Stewart, J. Q., \& Warntz, W. (1958). Physics of population distribution. Journal of Regional Science, 1(1), 99-121.

Turner, A. (2007). From axial to road-centre lines: A new representation for space syntax and a new model of route choice for transport network analysis. Environment and Planning. B, Planning \& Design, 34(3), 539-555.

van Nes, A., Berghauser-Pont, M., \& Mashhoodi, B. (2011). Combination of space syntax with Spacematrix and the mixed use index. In J. Greene, J. Reyes, \& A. Castro (Eds.), 8th international space syntax symposium. Chile: Santiago.

Waze (2015). Community-based mapping, traffic and navigation. www.waze.com. Accessed June 2015.

Webster, C. (2010). Pricing accessibility: Urban morphology, design and missing markets. Progress in Planning, 73(2), 77-111.

Wegener, M., \& Fürst, F. (2004). Land-use transport interaction: State of the art. Available at SSRN 1434678.

Yeh, A. G. O. (1999). Urban planning and GIS. In Geographical Information Systems: Principles, Techniques, Applications, and Management 2nd edition, Eds PA Longley, M Goodchild, D Maguire, D Rhind. John Wiley, New York, pp. 877-888.

Yeh, A. G. O. (1991). The development and applications of geographic information systems for urban and regional planning in the developing countries. International Journal of Geographical Information Systems, 5(1), 5-27. 